\title{
Little benefit from mesalazine taken prophylactically after surgery for Crohn's disease
}

Lochs H, Mayer M, Fleig WE, et al, and the European Cooperative Crohn's Disease Study VI Group. Prophylaxis of postoperative relapse in Crohn's disease with mesalamine: European Cooperative Crohn's Disease Study VI. Gastroenterology 2000;118:264-73.

\section{Question}

After surgery for Crohn's disease, does mesalazine taken prophylactically reduce the risk of clinical relapse?

\section{Design}

Randomised double-blind controlled trial with 18 months of treatment.

\section{Setting}

Twenty nine centres in Austria, Germany, Switzerland, and Scandinavia.

\section{Patients}

Three hundred and eighteen patients ( $51 \%$ women) aged 18-70 with Crohn's disease who underwent surgical resection, which in $75 \%$ was radical (that is, all macroscopic disease removed). Patients with a stoma or having had more than three previous operations were excluded. During the trial, treatment with immunosuppressive drugs, metronidazole, methotrexate, and other aminosalicylates was not allowed.

\section{Intervention}

Patients were allocated to mesalazine $4 \mathrm{~g}$ daily divided into three doses $(n=154)$ or placebo three times daily $(n=170)$, started within 10 days of surgery and continued for 18 months.

\section{Outcome measures}

The primary outcome was clinical relapse defined as either an increase in Crohn's disease activity index
(CDAI) to $>250$, a 60 point increase in CDAI to above 200 sustained for two weeks, need for surgery, new fistula development, or occurrence of a septic complication. Endoscopic relapse was a secondary outcome.

\section{Results}

Cumulative clinical relapse rate at 18 months was $24.5 \%$ in the mesalazine treated group versus $31.4 \%$ in the placebo treated group ( $\mathrm{p}=0.10$ by one sided log rank test). Study medication was discontinued by 49 patients in the mesalazine group and in 43 in the placebo group. Per protocol analyses did not differ from the results of the intention to treat analysis. In a subgroup analysis of those with isolated small bowel disease $(n=124)$, the relapse rate in mesalazine treated patients was $21.8 \%$ compared with $39.7 \%$ in placebo treated patients $(p=0.002$ by one sided log rank test). Endoscopic recurrence was assessed by colonoscopy and was available at 18 months for only 133 cases. After 18 months, $66 \%$ and $50 \%$ of the mesalazine and placebo groups, respectively, had evidence of endoscopic recurrence. No correlation was found between endoscopic findings and clinical relapse. Probability of clinical relapse was mainly influenced by disease duration, use of steroids in the six months preceding surgery, smoking habits, and age, and was unrelated to disease location. While minor adverse events were common in both groups, there were no serious adverse events that were clearly treatment related. No evidence of renal impairment was detected.

\section{Conclusion}

Prophylactic mesalazine for 18 months had no significant effect on the postoperative course of Crohn's disease overall but halved the relapse rate in a subgroup with isolated small bowel disease.

\section{Commentary}

The main disadvantage of surgery for Crohn's disease is postoperative recurrence of disease even when all macroscopically involved bowel is removed. Risk factors for early recurrence are ileal or ileocolonic resection with ileocolonic anastomosis, heavy smoking, especially in females, and perforating type of disease. Within weeks to months after resection, new lesions can be visualised endoscopically just proximal to the ileocolonic anastomosis that will later evolve to full blown Crohn's disease. The placebo group in controlled recurrence prevention trials yielded symptomatic recurrence rates of about $20 \%$ at one year and $40 \%$ at two years. Subsequently there is a $10 \%$ increase in symptomatic recurrence per follow up year.

Strategies to prevent recurrence of Crohn's disease after ileocolonic resection have been largely unsuccessful to date. The current trial by Loch et al is the largest trial to date using a 5-aminosalicylic acid (5-ASA) formulation for prophylaxis of Crohn's recurrence. All patients entered the study within 10 days after resection and received a high dose (4 g) of the sustained 5-ASA release formulation Pentasa. The overall results show clearly that 5-ASA formulations are not effective in preventing symptomatic recurrence up to 18 months after surgery. The positive results in the subgroup of patients with Crohn's disease involving the small bowel only before resection is somewhat surprising. Does Pentasa, which releases mesalazine in a sustained manner once entering the small bowel, deliver high enough concentrations of 5-ASA in the small bowel to exert its topical action in patients with limited colonic resection whereas patients with more extensive colonic resection have less mucosal availability for the drug? 


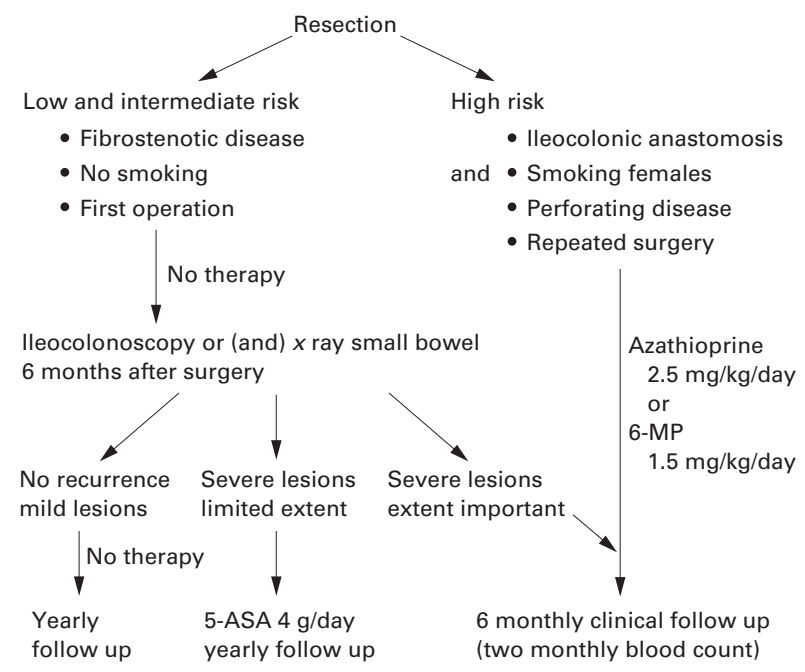

Figure 1 Algorithm for the prophylaxis of Crohn's recurrence after resection. 6-MP, 6-mercaptopurine; 5-ASA, 5-aminosalicylic acid.

Another explanation may be that patients operated on for small bowel disease alone have a more aggressive pattern of recurrence than patients with other locations and that the drug is particularly effective in those patients. However, no study has shown that patients with ileitis or ileocolitis behave differently after surgery. Caution must be observed because of the retrospective nature of the analysis as there may have been bias in the construction of the groups for analysis.

An important issue as far as interpretation of the negative data is concerned is patient compliance. Many patients do not take their drugs on a regular basis when they feel well, especially when the number of tablets they have to take is large. In the trial by Lochs et al, one third of patients violated the protocol but a per protocol analysis, excluding the protocol violators, did not change the outcome.

What do we learn from this report? Firstly, it is not advisable to give all patients operated on for Crohn's disease prophylactic therapy with 5-ASA. Although there may be a small benefit from giving 5-ASA, meta-analysis suggests that eight patients with ileal or ileocolonic resection would have to be treated with doses of up to 3-4 g/day for a period of 2-3 years at considerable cost to prevent one postoperative recurrence. ${ }^{1}$ Secondly, patients may not be prepared to take these large amounts of drugs long term to achieve at most a modest risk reduction in recurrence and the issue should be thoroughly discussed before embarking on this long term therapy.

Other prophylactic drug regimens are under study. Treatment with high dose metronidazole $(20 \mathrm{mg} / \mathrm{kg})$ and 6-mercaptopurine ( $50 \mathrm{mg} /$ day) is probably associated with better prophylactic potential than 5-ASA but only few studies have been reported. More controlled trials are needed but they should focus on high risk populations.

We believe it is better to individualise treatment. As symptomatic recurrence is preceded by tissue relapse, assessment of the bowel by endoscopy or $x$ ray 6-12 months after resection may allow selection of patients for intensive treatment. We propose the algorithm shown in fig 1 for the management of Crohn's disease postoperatively.

P RUTGEERTS

University Hospital Gashuisberg, Department of Internal Medicine, Herestraat 49, 3000 Leuven, Belgium Paul.Rutgeerts@uz.kuleuven.ac.be

1 Camma C, Giunta M, Roselli M, et al. Mesalamine in the maintenance treatment of Crohn's disease: a meta-analysis adjusted for confounding variables. Gastroenterology 1997:113:1465-73. 